\title{
Reassessment of the new diagnostic thresholds for gestational diabetes mellitus: an opportunity for improvement
}

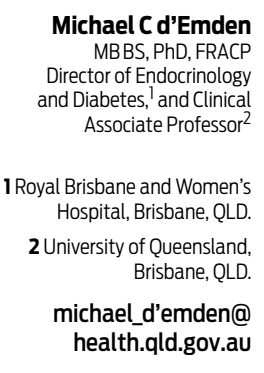

doi: 10.5694/mjal4.00277

\section{T}

he diagnosis of gestational diabetes mellitus (GDM) has been based on guidelines derived from expert opinion. The original threshold blood glucose levels (BGLs) for diagnosis recommended by the Australian Diabetes in Pregnancy Society (ADIPS) had no scientific validity. ${ }^{1}$ Subsequently, intervention studies provided validity for these levels. ${ }^{2,3}$ However, these criteria remained statistically unsupported until the results of the Hyperglycemia and Adverse Pregnancy Outcome (HAPO) study were published.

\section{Assessment of the Hyperglycemia and Adverse Pregnancy Outcome study}

HAPO analysed data from 23316 women enrolled in a blinded epidemiological study where neonatal and maternal adverse events were assessed according to prespecified blood glucose ranges after a $75 \mathrm{~g}$ oral glucose tolerance test performed at 24-32 weeks of gestation. ${ }^{4}$ This study showed strong continuous relationships between fasting blood glucose (FBG) levels, 1-hour BGL and 2-hour BGL after a $75 \mathrm{~g}$ oral glucose tolerance test and outcomes. HAPO assessed different odds ratios (ORs) for risk of developing adverse neonatal outcomes for each BGL parameter and the percentage of women diagnosed. Subsequently, the International Association of Diabetes and Pregnancy Study Groups (IADPSG) reached consensus that the arbitrary threshold level for risk should be an OR $\geqslant 1.75$ of developing certain adverse neonatal outcomes. These recommended levels were $\mathrm{FBG} \geqslant 5.1 \mathrm{mmol} / \mathrm{L}$, 1 -hour $\mathrm{BGL} \geqslant 10 \mathrm{mmol} / \mathrm{L}$ and 2 -hour $\mathrm{BGL} \geqslant 8.5 \mathrm{mmol} / \mathrm{L} .{ }^{5}$ The diagnosis is established if one or more BGLs equals or exceeds these values. If this threshold is met, a patient presumably will have at least a $75 \%$ increased risk of complications. These recommendations have been accepted by many professional bodies, including the American Diabetes Association. ${ }^{6}$ However, many other organisations have not endorsed them, including the United States National Institutes of Health (NIH). ${ }^{7}$ They have been recommended for endorsement in Australia by ADIPS. ${ }^{8}$ Based on these new criteria, there is a significant increase in the number of patients being diagnosed with GDM. In the HAPO study, $16.1 \%$ of participants met these criteria. ${ }^{5}$ The rate of GDM in different countries varies but there is a general concern that the IADPSG criteria will place an increased burden on services. ${ }^{9,10}$

This increase would not be an issue if these pregnancies were associated with a significant increase in preventable complications. But do these criteria identify pregnancies at risk? This may not be the case. There are at least two problems concerning the new diagnostic thresholds.

\begin{abstract}
Summary
The International Association of Diabetes and Pregnancy Study Groups has recommended new blood glucose levels (BGLs) for the diagnosis of gestational diabetes mellitus (GDM).

These BGLs supposedly identify women with at least a $75 \%$ increased risk of developing certain adverse neonatal outcomes.

The new criteria result in a significant increase in the number of women diagnosed with GDM.

- Most of the women diagnosed with GDM according to the new criteria have only one elevated BGL.

- Due to the unrecognised effect of the other BGLs being normal, up to $50 \%$ of these women are inappropriately diagnosed with GDM as they do not meet the agreed risk threshold.

In absolute terms, for every 100 women diagnosed with GDM who have only one elevated BGL, nearly 50 do not meet the agreed risk threshold for diagnosis, and there are only up to seven extra cases of large-for-gestationalage infants.

- A more statistically valid basis for diagnosing GDM consistent with the recommended risk threshold is suggested.
\end{abstract}

\section{The combined adverse neonatal outcomes}

The thresholds for diagnosis were based on the average BGLs at which the odds for birthweight $>90$ th percentile (measure of large for gestational age [LGA]), cord C-peptide levels $>90$ th percentile, and per cent body fat $>90$ th percentile reached 1.75 times the estimated odds of these outcomes at mean glucose values. There are several concerns with this approach. Considering the FBG threshold only, the calculated OR for the individual primary and secondary outcome measures of the HAPO study are shown in Box $1 .{ }^{5}$ The outcome measure of per cent body fat $>90$ th percentile was neither a primary nor secondary outcome measure but simply an exploratory outcome. There was incomplete ascertainment, with it being assessed in $83 \%$ of all babies and in only $73 \%$ of babies who had cord C-peptide levels measured. ${ }^{11}$ The data for this outcome were not published in the original results paper of HAPO. ${ }^{4}$ Including this outcome would not be considered standard statistical practice.

The primary outcome measure of cord C-peptide levels $>90$ th percentile was included but it is not a routine test performed in clinical practice. An increase in C-peptide levels at delivery most likely reflects the normal physiological increase in insulin secretion due to increased fetal glucose exposure consequent to maternal hyperglycaemia. ${ }^{4}$ It may be a marker of insulin resistance with relevance to future adverse metabolic outcomes, but it is not known if it has any long-term clinical significance. ${ }^{11,12}$ It was the major 


\begin{tabular}{|c|c|c|}
\hline Outcomes*† & OR & $95 \% \mathrm{Cl}$ \\
\hline \multicolumn{3}{|l|}{ Primary outcomes } \\
\hline Cord C-petide levels > 90th percentile & 2.02 & $1.85-2.21$ \\
\hline Birthweight $>90$ th percentile & 1.68 & $1.56-1.80$ \\
\hline Neonatal hypoglycaemia & 1.24 & $1.05-1.46$ \\
\hline Primary caesarean section & 1.18 & $1.11-1.26$ \\
\hline \multicolumn{3}{|l|}{ Secondary outcomes } \\
\hline Pre-eclampsia & 1.40 & $1.26-1.56$ \\
\hline Shoulder dystocia & 1.30 & $1.07-1.58$ \\
\hline Preterm delivery & 1.16 & $1.05-1.28$ \\
\hline Hyperbilirubinaemia & 1.00 & $0.92-1.09$ \\
\hline Neonatal intensive care & 0.99 & $0.91-1.08$ \\
\hline \multicolumn{3}{|c|}{$\begin{array}{l}\text { OR = odds ratio. * Fully adjusted calculated ORs ( } 95 \% \text { Cls) at the } \\
\text { FBG threshold; adapted from International Association of Diabetes } \\
\text { and Pregnancy Study Groups Consensus Panel (online Appendix). }{ }^{5} \\
\text { †Neonatal adiposity > 90th percentile is not shown as it was not a } \\
\text { prespecified primary or secondary outcome measure.1" }\end{array}$} \\
\hline
\end{tabular}

outcome measure contributing to the lower FBG threshold (Box 1): its OR is 2.02, significantly higher than for adiposity $(\mathrm{OR}, 1.62)$ and LGA $(\mathrm{OR}, 1.68)$. If increased numbers of women are being diagnosed with GDM because they are at risk of their fetus having high cord C-peptide levels but not LGA, the significance of this abnormality should be established for it to be included in the combined outcome measure.

The combination of these outcome measures appears to have been a post-hoc decision made by IADPSG. These were selected because they had had the greatest difference between the at-risk group and the normal group. Neonatal adiposity $>90$ th percentile was not a prespecified combined outcome measure. ${ }^{4}$ This should be considered as an exploratory combined outcome measure at best. Its validity should be proven in other cohorts. It is inappropriate to use it as the basis for establishing new diagnostic thresholds for GDM.

There were four primary outcome measures originally defined for HAPO (Box 1). A more statistically valid approach would be to combine all the four primary outcome measures. This was not done, so the highly relevant clinical primary outcome measures of rates of caesarean section or neonatal hypoglycaemia did not contribute to the diagnostic thresholds. If this had been undertaken, higher BGLs would have been defined to achieve the OR threshold of 1.75 , with fewer women identified.

The most recognised clinical problem of gestational diabetes is macrosomia. ${ }^{1-5}$ The outcome measure assessing this in HAPO was birthweight $>90$ th percentile. At the threshold FBG level of $5.1 \mathrm{mmol} / \mathrm{L}$, the OR for LGA is 1.68 (95\% CI, 1.56-1.80) (Box 1). This implies that the mean risk for LGA at this FBG level is below the IADPSG threshold for diagnosis. A higher FBG level is required to reach the defined OR. Ideally, to ensure with $95 \%$ confidence that women diagnosed with GDM are at risk, the lower limit of the $95 \%$ confidence interval of the OR for each BGL should be equal to or above 1.75 . These values cannot be calculated from the available data.

\section{The effect of normal blood glucose levels}

The second problem relates to the effect of normal BGL parameters on risk. When the IADPSG-recommended thresholds are used to diagnose GDM in the blinded cohort of HAPO, $69 \%$ of women meet the criteria based on a single elevated BGL parameter only. ${ }^{13}$ Of these women, $81 \%$ were diagnosed on the basis of an elevated FBG or 1-hour BGL. ${ }^{13}$ The effect on overall risk of having two normal BGLs in these women was not considered. An exploratory analysis assessing this effect suggested that potentially up to $50 \%$ of these women may not reach the required risk threshold. ${ }^{14}$ In response, data from HAPO were reported for women who had only one, two or three elevated BGLs (Box 2). ${ }^{13}$ The mean OR for LGA of women with an elevated FBG or 1-hour BGL only was 1.76 and the $95 \%$ CI crossed the risk threshold, confirming that nearly $50 \%$ of these women did not meet the agreed risk threshold. This implies that overall nearly one-third (about 50\% of the 69\%) of women who had only one elevated BGL did not meet the risk threshold. This also applies to subjects with two elevated postprandial BGLs only (Box 2). Most of the potential increase in clinical demand results from patients who do not meet the IADPSG agreed level of risk for diagnosis.

In contrast, women with an elevated FBG level (a marker of preprandial hyperglycaemia) plus one or more elevated post-challenge BGLs (markers of postprandial hyperglycaemia) have pregnancies at much greater risk of LGA (mean OR, >3.0; lower limit of the 95\% CI, >2.0; Box 2). These elevated BGLs suggest greater intrauterine exposure to glucose associated with higher rates of LGA compared with women who had one elevated BGL only. These data also suggest that a lower FBG level in combination with a lower 1-hour or 2-hour BGL (or both) may still identify patients at risk.

In absolute terms, for every 100 women in HAPO diagnosed with GDM using the new IADPSG criteria who have only one elevated BGL, nearly 50 do not meet the agreed risk threshold. In these pregnancies, most cases of LGA are not due to GDM. ${ }^{13}$ Intervention in these pregnancies may not change the rate of caesarean delivery but the rate of induction may be increased. More babies born to women labelled with GDM will be admitted to specialised neonatal nurseries, but with no decrease in the rate of neonatal hypoglycaemia. ${ }^{2,3}$ It is not known whether any of the secondary benefits of intervention will apply to this cohort given the low rate of LGA attributable to GDM. It is not known whether these women are at increased long-term risk of developing type 2 diabetes (T2DM).

\section{Discussion}

This assessment suggests that the new diagnostic criteria are based on a questionable composite end point, resulting in more women being diagnosed with GDM. This increase in numbers is predominantly due to women having a minimally elevated FBG level or 1-hour BGL only. Many of these women do not reach the agreed arbitrary risk threshold for diagnosis. Their long-term risk of T2DM is unknown.

There are many consequences of this. Initially, GDM was based on the risk of developing T2DM. ${ }^{15}$ It is recommended that women with GDM are assessed regularly 
2 Risk of large-for-gestational-age (LGA) birthweight among women with one, two or three elevated blood glucose levels (BGLs) in the Hyperglycemia and Adverse Pregnancy Outcome (HAPO) study*

All BGL $\geqslant$ thresholds

1-hour and 2-hour BGL elevated only

FBG and 2-hour BGL elevated only

FBG and 1-hour BGL elevated only

2-hour BGL $\geqslant 8.5 \mathrm{mmol} / \mathrm{L}$ only

1-hour BGL $\geqslant 10.0 \mathrm{mmol} / \mathrm{L}$ only

$\mathrm{FBG} \geqslant 5.1 \mathrm{mmol} / \mathrm{L}$ only

All BGL $<$ thresholds
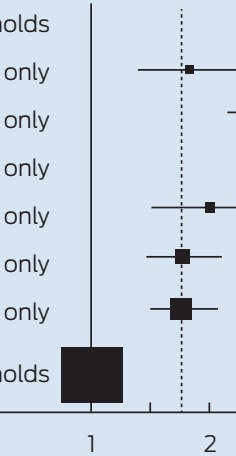

2

3

4

5

Odds ratio

FBG = fasting blood glucose. $*$ The odds ratio (OR) and 95\% confidence intervals for LGA by BGL subgroups in the HAPO study are adapted from Metzger and Dyer. ${ }^{13}$ Also shown is the International Association of the Diabetes and Pregnancy Study Groups OR risk threshold of 1.75 for diagnosis of GDM (dotted line).

for the development of diabetes, resulting in increased long-term costs and inconvenience, especially if they are not actually at risk. Additionally, women may be charged higher life insurance premiums because of a prior diagnosis of GDM.

It is reported ${ }^{16}$ that the NIH consensus panel has recommended as a priority that a new randomised controlled trial is conducted that evaluates outcomes in women currently classified as "normal" according to current US criteria but meeting IADPSG ${ }^{5}$ or American Diabetes Association criteria. ${ }^{6}$ A similar study is suggested for Australia. (William Hague, Head, Obstetric Medicine Group, School of Paediatrics and Reproductive Health, University of Adelaide, personal communication, June 2014.) If these studies enrol women who have only one minimally elevated BGL, there will be fewer adverse events than anticipated with little chance of demonstrating benefits from intervention. Large sums of money and investigator time and effort may be wasted undertaking these trials.

The risk threshold for diagnosis is an arbitrary decision. It has been suggested that the OR threshold could be raised to 2.0 to reduce patient numbers. ${ }^{17}$ If this threshold is based on the same combined outcome measure, and no adjustment is made for the effect of normal BGLs on risk, the same problems discussed here will arise. This would be unnecessary if an improved diagnostic approach is adopted. Improved identification of at-risk patients could justify adopting a higher OR threshold.

The most clinically recognised complication of GDM is macrosomia, assessed in HAPO as birthweight > 90th percentile (LGA). This analysis suggests that women who have only one elevated BGL parameter require a higher threshold to ensure with $95 \%$ confidence that they meet the agreed risk threshold. ${ }^{5}$ Multiple diagnostic rules based on whether a patient has one, two or three BGLs exceeding different threshold levels would overcome this problem but would be unduly complicated. Alternatively, a risk calculator based on the HAPO data could be an excellent solution. However, the approach recommended by
IAPDSG is compellingly simple and a modification of this would seem to have merit.

\section{Recommendation}

A statistically justified strategy would be to make some minor modifications to the IADPSG criteria. The currently recommended IADPSG criteria correctly identify most patients with two or more elevated BGLs, including the FBG level. In fact, lower FBG level and post-challenge BGLs in combination could be justified, as the mean OR was significantly elevated above the threshold (mean OR, $>3.0$ for all subgroups; lower limit of the 95\% CI, > 2.0; Box 2). To establish the diagnosis on the basis of only one elevated BGL, the thresholds must be higher than the currently recommended IADPSG levels to correctly identify women at risk. These thresholds should equate to the lower limit of the $95 \%$ CI corresponding to an OR of 1.75 for LGA. This approach would more accurately identify these patients.

\section{Competing interests: No relevant disclosures.}

Provenance: Not commissioned; externally peer reviewed.

1 Martin Fl. The diagnosis of gestational diabetes. Ad Hoc Working Party. Med J Aust 1991; 155: 112

2 Crowther CA, Hiller JE, Moss JR, et al. Effect of treatment of gestational diabetes mellitus on pregnancy outcomes. N Engl J Med 2005; 352: 2477-2486.

3 Landon MB, Spong CY, Thom E, et al. A multicenter, randomized trial of treatment for mild gestational diabetes. N Engl J Med 2009; 36l: 1339-1348.

4 HAPO Study Cooperative Research Group, Metzger BE, Lowe LP, et al. Hyperglycemia and adverse pregnancy outcomes. NEngl J Med 2008; 358: 1991-2002.

5 IADPSG Consensus Panel. International Association of Diabetes and Pregnancy Study Groups recommendations on the diagnosis and classification of hyperglycemia in pregnancy. Diabetes Care 2010; 33 : 676-682.

6 American Diabetes Association. Standards of medical care in diabetes 2011. Diabetes Care 2011; 34 Suppl 1: S11-S61.

7 National Institutes of Health Consensus Development Conference. Diagnosing gestational diabetes mellitus. 2013. http://consensus.nih. gov/2013/gdmstatement.htm (accessed Jun 2014).

8 McIntyre DH, Oats JJ. Gestational diabetes needs to be managed. Med J Aust 2013; 198: 78-79.

9 Moses RG, Morris GJ, Petocz P, et al. Impact of potential new diagnostic criteria on the prevalence of gestational diabetes mellitus in Australia. Med J Aust 2011; 194: 338-340.

10 Sacks DA, Hadden DR, Maresh M, et al. Frequency of gestational diabetes mellitus at collaborating centers based on IADPSG consensus panelrecommended criteria: the Hyperglycemia and Adverse Pregnancy Outcome (HAPO) Study. Diabetes Care 2012; 35: 526-528.

11 HAPO Study Cooperative Research Group. Hyperglycemia and Adverse Pregnancy Outcome (HAPO) Study: associations with neonatal anthropometrics. Diabetes 2009; 58: 453-459.

12 Boney CM, Verma A, Tucker R, Vohr BR. Metabolic syndrome in childhood: association with birth weight, maternal obesity, and gestational diabetes mellitus. Pediatrics 2005; 115: e290-e296.

13 Metzger BE, Dyer AR. Comment on d'Emden. Do the new threshold levels for the diagnosis of gestational diabetes mellitus correctly identify women at risk? Diabetes Care 2014; 37: e43-e44. doi: 10.2337/dc13-2526.

14 d'Emden M. Do the new threshold levels for the diagnosis of gestational diabetes mellitus correctly identify women at risk? Diabetes Care 2014; 37 : e30. doi: 10.2337/dcl3-2234.

15 O'Sullivan JB, Mahan CM. Criteria for the oral glucose tolerance test in pregnancy. Diabetes 1964; 13: 278-285.

16 Mclntyre HD. Diagnosing gestational diabetes mellitus: rationed or rationally related to risk? Diabetes Care 2013; 36: 2879-2880.

17 Manzoor N, Moses RG. Diagnosis of gestational diabetes mellitus: a different paradigm to consider. Diabetes Care 2013; 36: el87. doi: 10.2337/ dcl3-1433. 